\title{
Romulus comme altellus (Verrius Flaccus, Ovide): Sémantique et portée symbolique
}

\author{
Vincent Martzloff - Barbora Machajdíková \\ (Sorbonne University, Paris; Comenius University, Bratislava)
}

\section{Romulus as altellus (Verrius Flaccus, Ovid): Semantics and symbolic significance}

\begin{abstract}
Romulus and Remus were twin brothers, but according to a curious statement by John Lydus, Romulus was younger than Remus. The gloss altellus Romulus dicebatur transmitted by Paul the Deacon suggests that the Byzantine scholar may have been preserving an old tradition. Although the form altellus has been interpreted in various ways, the likeliest explanation is that altellus goes back to *alter-elo-s and means 'the second of the twin brothers'. Of the twins, Remus was the first to be born. The word altellus is not related to altus 'high, tall' or alere 'nourish', despite the attempts of ancient authorities (and more recently the Christian cabalist Egidio da Viterbo) to buttress such an etymology. Moreover, a playful allusion to altellus can possibly be found in Ovid's Fasti (Romulus alter erit), which would confirm the reconstruction *alter-elo-s. As a result, the founder of Rome is not the elder brother. Scholars have emphasised that the biblical twins Esau and Jacob offer a close parallel, as Jacob, who may be regarded as the founder of Israel, was not the firstborn son. The parallel is even more striking if one pays attention to the fact that in the Book of Jubilees and the Testaments of the Twelve Patriarchs, Esau was killed by his brother. The logic of these stories is ultimately based on a high degree of diagrammaticity or constructional iconicity between the order of the twins' births and their personal tropism: Esau (a hunter) and Remus are associated with a quite primitive way of life (elder brother: PAST), whereas Jacob and Romulus are characterised by metis and are presented as the bearers of a more advanced way of life (younger brother: MODERNITY). The word altellus is rich in implications because it encapsulates the fact that the elder brother must be supplanted by his younger twin.
\end{abstract}

\section{Keywords}

Book of Jubilees; Egidio da Viterbo; Festus; foundation of Rome; Jacob and Esau; John Lydus; laughter; Ovid; Quirinus; Remus; Romulus; twins 


\section{Les traditions de Verrius Flaccus sur altellus et leurs échos}

Le mot altellus est attesté chez Paul Diacre, l'abréviateur de Festus qui résume lui-même l'œuvre de l'érudit augustéen Verrius Flaccus: ${ }^{1}$ Altellus Romulus dicebatur, quasi altus in tellure, uel quod tellurem suam aleret; siue quod aleretur telis; uel quod a Tatio Sabinorum rege postulatus sit in conloquio pacis, et alternis uicibus audierit locutusque fuerit. Sicut enim fit diminutiue a macro macellus, a uafro uafellus, ita ab alterno altellus (Paul. Fest. 6, 29-7, 4 L) 'altellus était le nom donné à Romulus, comme s'il avait été nourri / élevé (altus) sur la terre (in tellure), ou parce qu'il nourrissait / faisait croître (aleret) sa propre terre (tellurem), ou parce qu'il se nourrissait / parce qu'il était élevé (aleretur) par les armes (telis), ou parce que, sollicité par Tatius, le roi des Sabins, dans des pourparlers en vue de la paix, il avait écouté et pris la parole tour à tour (alternis uicibus). En effet, de même que macer (maigre) a pour diminutif macellus et uafer (habile) uafellus (finaud), pareillement alternus a pour dérivé altellus.'

Le mot est aussi transmis par des gloses (parfois avec des déformations): altellus altus diminũ (CGL 2, 565, 51), altellus terra nutritus (CGL 4, 206, 10), altellus terre nutritus (CGL 5, 438, 54), alcitellus alte uocatus (CGL 5, 491, 6), alcitellus alte euocatus (CGL 4, 206, 8), acitella alte edocatus (CGL 5, 436, 17). On ajoutera altellum hoc est terrenum (Löwe 1875: p. 12; Hagen 1876: p. 340). Sur l'ensemble, voir Warren (1884: p. 131) et Peruzzi (1967: p. 367).

Pour nous, le mot altellus est un hapax ou un quasi hapax. Mais, comme le remarque Peruzzi (1967), la multiplicité des explications étymologiques enregistrées par Verrius Flaccus démontre que altellus était un terme sur lequel les érudits romains avaient probablement beaucoup réfléchi dans plusieurs de leurs travaux.

On trouve chez Ovide une possible allusion aux réflexions des antiquaires relatives à altellus, dans un passage des Fastes consacré à une première étymologie des mots Lupercal et lupercus (Fast. 2, 381-422). L'oncle de la vestale Silvia donne l'ordre de noyer les nouveau-nés dans le Tibre (in amne necari, 385). Le souverain fait alors l'objet d'une apostrophe, à la deuxième personne du singulier: Quid facis? Ex istis Romulus alter erit (Fast. 2, 386). Schilling (1993a: p. 43) traduit 'Que fais-tu là? L'un de ceux-ci sera Romulus!'. On peut se demander si l'énoncé rapporté au vers 386 est à attribuer au narrateur lui-même ou aux serviteurs du roi (ministri, 387) qui, dans un premier temps, rejettent les ordres qui leur sont donnés (iussa recusantes). Selon M. Ver Eecke, le choix opéré par Ovide de juxtaposer Rōmulus et alter pourrait s'interpréter comme un jeu littéraire fondé sur une référence discrète aux débats savants concernant la signification de altellus. ${ }^{2}$ Si cela est le cas, on doit admettre qu'il existait une conception herméneutique d'après laquelle le mot altellus était expliqué non par le verbe alere, ni par l'adjectif alternus, mais par alter. Surtout, le passage ovidien est important parce que le contexte suggère que altellus est à mettre en relation avec une situation liée à la naissance ou à la petite enfance de Romulus,

1 Sur Verrius Flaccus et ses abréviateurs, on consultera Lhommé (2011). Les textes de Festus et de Paul Diacre sont cités d'après l'édition de Lindsay (1913).

2 En ce sens, Ver Eecke (2008: p. 198, note 29; 2010: p. 30). 
non à un quelconque rapport avec Titus Tatius (comme pourrait le faire croire l'une des interprétations enregistrées par Paul Diacre). ${ }^{3}$

La poétique ovidienne est coutumière de telles remotivations sémantiques, que le chantre de Sulmone applique volontiers au fondateur de l'Urbs. Ainsi, dans les paroles que Junon destine à Hersilia, la femme de Romulus divinisé (Met. 14, 833-834), Ovide réactualise, par un brillant jeu d'allusion, l'étymologie du théonyme Quirinnus (issu de *ko-wir-ino-s), ${ }^{4}$ composé contenant le lexème uir (toutefois, bien sûr, non au sens de héros ou d'homme tenant lieu d'époux, mais de membre de la communauté civique): dignissima tanti \# ante fuisse UIR / / coniunx nunc esse QUIRini 'très digne d'avoir été auparavant l'épouse d'un si grand homme, d'être à présent celle de Quirinus'. Les mots uirī et Quirin̄in, réunis sur l'axe syntagmatique, sont également associés sur l'axe paradigmatique. ${ }^{5}$ La remotivation 'maritale' de Quirīnus est même double, puisqu'elle porte non seulement sur uir, mais aussi sur le préfixe co-(*ko-wir-ino-s), dont la présence latente est revivifiée par con-iunx. Poète érudit, Ovide avait selon nous pleinement conscience de la morphologie de Quirinus et a détourné avec humour le sens originel de ce théonyme: le dieu de la communauté civique est ici devenu un dieu-conjoint.

À la Renaissance, la glose de Paul Diacre a retenu l'attention de l'humaniste et kabbaliste chrétien Gilles de Viterbe (Egidio da Viterbo, 1469-1532), qui fut aussi cardinal. Dans la Scechina (Secret 1959: p. 118-119), l'auteur refuse le rattachement traditionnel du toponyme Rōma au substantif grec $\hat{\rho} \omega \dot{\mu \eta \eta}$ 'force' pour proposer une explication à partir du mot hébreu rāmā 'position élevée, hauteur, éminence'. Il se fonde pour cela sur la glose de Paul Diacre Altellus Romulus dicebatur, quasi altus in tellure qu'il comprend comme 'Romulus was called Altellus, as if tall on the earth' (Stein Kokin 2011: p. 36). Mais cette interprétation de altus n'est apparemment pas la meilleure dans le contexte de la glose. ${ }^{6}$ Gilles de Viterbe croyait trouver une confirmation de son étymologie chez Virgile (altae moenia Romae, Aen. 1, 7). Altellus (que Gilles dérive de altus 'haut') serait donc dans son esprit, semble-t-il, une sorte de traduction du nom Rōmulus dont l'origine serait à chercher dans la langue des Hébreux ( a lingua mea).

3 Un autre passage des Fastes (2, 485-486) est lui aussi construit autour de alter, appliqué cette fois au jumeau défunt. Mars réclame l'apothéose pour Romulus (redde patri natum): même si son autre fils a disparu (quamuis intercidit alter), l'enfant qui lui reste (qui mihi restat) occupera au ciel la place des deux frères (pro se proque Remo [...] erit). La présence de erit à la fin des vers 386 et 486 pourrait suggérer que l'un des deux passages a inspiré la rédaction de l'autre.

4 Cette analyse diachronique (acceptée par la plupart des chercheurs) est confirmée par Uirìtēs Quirinī (Gell. 13, 23, 2). [La quantité du premier $i$ peut faire débat, car Uiritês a pu être extrait de Quiritês, mais avoir ensuite été influencé par uīrēs. $]$ L'évolution phonétique $[\mathrm{kow}]>[\mathrm{kuw}]>[\mathrm{k} . \mathrm{w}]>\left[\mathrm{k}^{\mathrm{w}}\right]$ (labiovélaire secondaire, notée par le digramme $q u)$ présente une fermeture $[\mathrm{o}]>[\mathrm{u}]$ en syllabe prétonique comparable (selon nous) à la fermeture [e] > [i] de Minerua <*menes-wā. Particularité liée aux apostrophes (invocations) et aux tours exclamatifs du type équirine (iusiurandum per Quirinum, Paul. Fest. 71, 17 L)? Même phénomène dans Quiritês, où la réduction proviendrait des emplois au vocatif.

5 On sera attentif à l'extrême élaboration formelle du passage: $t A N T i / A N T e$, dignISSima / fuISSE / eSSE, coniUNCs / nUNC, position de uirì devant la césure, figure étymologique entre le superlatif de dignus (< *dekno-s avec la racine de decet) et le decus qui précède, paire minimale morphologique fuisse / esse ("polychrone').

$6 \quad$ Ver Eecke (2008: p. 198) traduit 'comme s’il avait été élevé sur terre'. Briquel (2018: p. 185) retient ici la traduction de altus par 'haut' ('en tant que haut (altus) sur la terre'). 


\section{Romulus comme altellus, le 'deuxième' des jumeaux?}

Quelle analyse diachronique doit-on accepter pour altellus? Lindsay (1891: p. 9), influencé par le rapprochement avec alere qu'on trouve chez Paul Diacre, interprétait altellus comme 'the little nursling' (par référence à l'allaitement de la louve). Mais un tel qualificatif ne définit pas Romulus de façon spécifique et pourrait s'appliquer aussi à Rémus. Selon Weinstock (1933: p. 150), le nom Rōmulus était rattaché à rūma 'mamelle, pis' dans l'esprit de certains locuteurs latinophones, ce qui justifierait un apparentement de altellus avec le verbe alere (cf. Nogara 1916). Mais ce raisonnement n'apprend rien sur l'étymologie réelle de altellus, car la connexion de altellus avec alere repose probablement sur une réinterprétation secondaire du mot par les locuteurs et n'a pas plus de valeur que la mise en rapport de Rōmulus avec rūma: le nom Rōmulus n'est évidemment pas dérivé de rūma, mais de Rōma.

Une meilleure solution a été proposée par Kretschmer (1909: p. 303), qui interprète altellus comme le diminutif de alter. L'analyse étymologique de Kretschmer a été acceptée par Peruzzi (1967), Briquel (1980: p. 268, note 7), Wiseman (1995: pp. 94, 102, 203) et Carandini (2006: p. 86). En revanche, l'interprétation de Kretschmer a été rejetée par Weinstock (1933: p. 150), mais sans contre-argument décisif. Peruzzi reconstruit correctement *alter-(e)lo-s et rejette *altern-(e)lo-s. Kretschmer (1909: p. 303) posait *alter-lo-s. Nous restituons *alter-elo-s (en supposant une syncope du $*[\mathrm{e}]$ et une assimilation subséquente $*[\mathrm{rl}]>[11])$.

La question essentielle est de déterminer par rapport à qui (et en quoi) Romulus a été qualifié de altellus. Nous écartons l'idée de Peruzzi (1967) qui supposait que altellus s'appliquait à Romulus par référence à Titus Tatius, qui, selon Peruzzi, aurait été le vainqueur dans la guerre qu'il avait entreprise contre les Romains. Malgré Peruzzi, rien ne prouve que, lors de leur règne commun, Romulus ait reçu une position subordonnée par rapport à Titus Tatius. Et on n'a pas d'arguments suffisants pour admettre que altellus signifiait 'successore' (Rocca 2005: p. 95). D'autres pistes furent prises en compte par Kretschmer (1909: p. 303): 'So dunkel es bleibt, wo und wie diese Benennung gebraucht war, bezeichnet sie doch deutlich den Romulus als den Anderen, den Zweiten, ob dies nun als alter frater, alter gemellus oder alter conditor zu verstehen ist'.

Pour établir la motivation sémantique de altellus, on pourrait vouloir recourir à des données onomastiques comparatives, mais celles-ci ne semblent pas être d'un grand secours. Si on suppose que le terme altellus exprime un contraste entre le futur conditor et son frère, comme l'admettent Kretschmer (1909) et Briquel (2013: p. 79), on pourrait songer à confronter altellus au nom gréco-phrygien $\Delta$ oías désignant le frère de

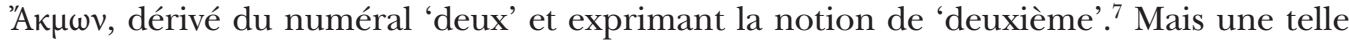
comparaison avec $\Delta$ oíaৎ ne permet pas d'élucider le sens précis de altellus et reste peu pertinente.

7 Les frères sont cités dans les Ethnica d'Étienne de Byzance (Billerbeck \& Zubler 2011: p. 52-53) et dans les scholies à Apollonios de Rhodes (2, 373, cf. Lachenaud 2010: p. 256-257). Bibliographie dans Martzloff (2015-2016: pp. 150-151). 
Puisque altellus dérive de alter contenant un suffixe -ter à valeur différenciative, ${ }^{8}$ on songe à faire intervenir les Nartes Exsar et Exserteg, qui sont des jumeaux (fazzctte), et

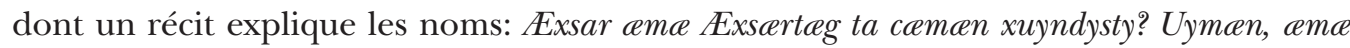
Exsar, xister afsymar, uyd cexsardžyn, Exsarteg ta, kester afsymar - Exsarcj axsardžyndcer. 'Pourquoi avaient-ils été appelés Exsar et Exserteg? Parce qu'Exsar, le frère aîné (хистæр), était brave (æхсарджын) et Axsarteg, le frère cadet (кæстæр), était encore plus brave (æхсарджындæр) qu'Axsar.' Mais les noms ossètes expriment une qualité (la bravoure), de façon simple ou relative, tandis que altellus exprime simplement un rang. Le parallèle est donc imparfait.

Si on souhaite comprendre le sens attaché à altellus, il faut distinguer cinq questions différentes, qui ont trop souvent été mêlées dans les études précédentes, et qui n'appellent pas nécessairement de réponse unitaire: (1) la question de la priorité rhétorique dans les syntagmes \{Remus, puis Rōmulus\} dans cet ordre; (2) la substitution de Rémus à Romulus dans des métonymies désignant Rome ou les Romains; (3) la question de la possible antériorité de Rémus par rapport à Romulus dans une phase ancienne de la légende et la possibilité que les jumeaux aient porté d'autres noms; ${ }^{9}$ (4) la question de la priorité de l'âge (lequel des jumeaux est né en premier?); (5) la question complexe (mais cruciale) de la primauté de l'un des jumeaux sur l'autre dans la structure narrative de l'histoire, où tantôt Rémus a (ou semble avoir) la prééminence sur Romulus, tantôt c'est Romulus qui a la prééminence sur son frère.

Rémus bénéficie parfois d'une priorité d'ordre rhétorique (Kretschmer 1909: p. 303; Wiseman 1995: p. 203; Briquel 2013: p. 78). Naevius avait composé une tragédie intitulée Alimonium Remi et Romuli. ${ }^{10}$ Cet ordre est attesté chez Cassius Hemina (fr. 14 de Chassignet 1999: p. 6, Remum et Romulum), chez Varron cité par Festus (332, 10 L, Remo et Romulo, cf. Paul. Fest. 333, 5 L), chez Cicéron (Leg. 1, 8, de Remo et Romulo, formule proverbiale à en juger par l'expression ut aiunt qui précède), dans les Fastes de Préneste (Remi et Rom[uli, 23 décembre), chez Tacite (Ann. 13, 58, Remi Romulique), chez Justin (43, 2, 7, alteri Remo, alteri Romulo). Il serait probablement erroné d'expliquer l'ordre \{Remus, puis Rōmulus\} comme une simple licence poétique. On pourrait certes renverser la perspective et faire valoir que l'ordre \{Remus, puis Rōmulus\} correspond à une tendance rythmique naturelle consistant à placer en seconde position l'élément dont le volume syllabique est le plus lourd (règle des membres croissants), tandis que l'ordre pour nous habituel $\{$ Rōmulus, puis Remus\} tient simplement au fait que Romulus a le premier rang parce qu'il est le fondateur. Mais cela expliquerait mal Remus et Romus (Serv. Aen. 1, 273, cf. Bruggisser 1987: p. 280; Myth. Vat. 1, 30, 2), à moins de supposer

8 Voir Martzloff (2013: p. 119), avec références bibliographiques.

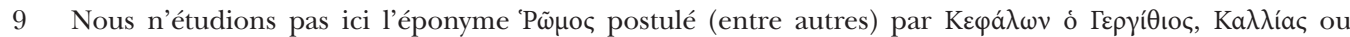

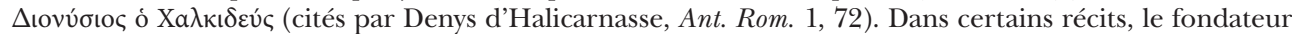

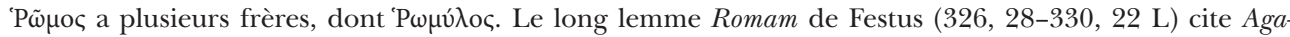
thocles qui faisait de Rhōmus le fondateur de Rome. On consultera Kretschmer (1909) et Ver Eecke (2008: pp. 193-194).

10 Don. ad Ter. Ad. 537 (4, 1, 21). Cette tragédie à sujet romain est-elle identique au Romulus cité par Varron (ling. 7, 54, 107) et au Lupus cité par Festus (334, 9 L), peut-être valant lupus femina? Voir Wiseman (1998: p. 3). 
que le [ō] long ait joué un rôle. En fait, même si l'ordre \{Remus, puis Rōmulus\} peut avoir une motivation plus profonde, il n'est pas prouvé que cet agencement des noms propres soit directement pertinent pour la problématique de altellus. Nous n'incluons donc pas cette donnée dans notre raisonnement, afin d'éviter qu'un argument mal assuré n'ait pour effet d'affaiblir la démonstration.

La substitution de Remus à Rōmulus (ou la mention exclusive de Remus au lieu des deux frères) pourrait certes suggérer que Rémus était le jumeau principal (mais que faudrait-il entendre par 'principal'?) dans un état ancien de la légende: Remi nepotes (Catull. 58, 5), signa Remi (Prop. 4, 6, 80), regnaue prima Remi (Prop. 2, 1, 23), turba Remi (Iuv. 10, 73),

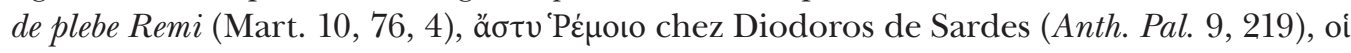

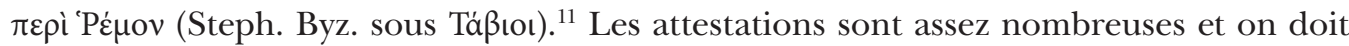
donc hésiter à les expliquer comme de simples licences poétiques. Or, dans les versions classiques de l'histoire des jumeaux albains, l'Urbs a été fondée par un unique ${ }^{12}$ personnage, et ce conditor unique ne s'appelle pas Remus, mais Rōmulus. Il est possible que la mention exclusive de Rémus et l'ordre \{Remus, puis Rōmulus\} représentent deux facettes d'un même phénomène. Malheureusement, toute tentative pour expliquer les mentions du seul Rémus risque de rester spéculative. ${ }^{13}$ En tout cas, nous ne pensons pas que les formules du type Remi nepotes soient directement pertinentes pour la compréhension du sens de altellus (contrairement à ce qui est généralement supposé par les chercheurs). Comme le montrera la suite de notre raisonnement, la signification profonde du mot altellus représente probablement un problème entièrement distinct de la question (certes importante en elle-même) de l'origine des expressions comme Remi nepotes.

11 Voir Billerbeck \& Neumann-Hartmann (2016: p. 248), Kretschmer (1909: p. 303, note 2).

12 Quand Tite-Live $(10,23,12)$ écrit simulacra infantium conditorum urbis sub uberibus lupae posuerunt, il s'agit d'une expression soit abusive, soit non conforme à la vulgate.

13 Il est bien sûr concevable que les noms des jumeaux (en particulier celui du fondateur) aient changé au cours du temps. En raison de sa transparence même, le nom propre Rōmulus se dénonce comme un dérivé du toponyme Rōma. Le nom originel du fondateur a peut-être été rayé et remplacé. En outre, le nom Rōmulus a dû être créé seulement au moment où l'Urbs naissante a reçu le nom de Rōma. Or, selon plusieurs chercheurs, il n'est pas sûr que la 'ville' fondée sur le Palatin ait porté le nom de Rōma dès le moment de sa fondation. Il existe peut-être même des indices (certes ténus et controversés) qui invitent à penser le contraire. S'appuyant sur le nom de la Porta Rōmāna ou Rōmānula (Varr. ling. 5, 164), Prosdocimi (2010: pp. 477-484) a suggéré que le nom Rōma pouvait être un micro-toponyme préexistant, référant à un lieu-dit qui était immédiatement voisin de, mais extérieur à la ville primitive, et que ce lieu-dit a été intégré à la ville dans un second temps et lui a imposé son nom. [Mais on pourrait aussi bien faire l'hypothèse inverse: rien n'interdirait de penser que Rōma ait pu être à l'origine un micro-toponyme désignant un lieu-dit situé à l'intérieur du Palatin à proximité de cette porte, et que ce micro-toponyme ait donné son nom d'abord à cette porte et ensuite à l'ensemble de l'espace 'urbain' délimité.] Dans cette perspective, on ne peut pas écarter l'idée que, dans une version ancienne de l'histoire des jumeaux renvoyant à ce stade premier, les frères aient porté les noms Remus et $X$ (Remus étant le nom du conditor) et qu'ensuite, dans un récit faisant allusion au stade ultérieur où la ville s'appelait désormais Rōma, le conditor ait reçu le nom transparent (c'est-à-dire linguistiquement motivé) de *Rōmos ou *Rōmelos (d'où Rōmulus), de sorte que le nom Remus s'est trouvé mécaniquement déclassé et a remplacé le nom $X$ de son jumeau par un simple effet de décalage. Dans cette perspective, les expressions du type Remi nepotes feraient référence à l'état ancien, dont le souvenir se serait conservé marginalement. Nous n'insistons pas sur ce point, qui est étranger à la problématique du mot altellus qui seule nous occupe ici. Et nous ne voulons nullement affirmer qu'une telle reconstitution doive être la bonne. Enfin, nous rappelons que l'argument de la Porta Rōmānula a été contesté par Grandazzi (1998). 
Rémus aurait eu la priorité par l'âge. Il existe une tradition d'après laquelle Romulus

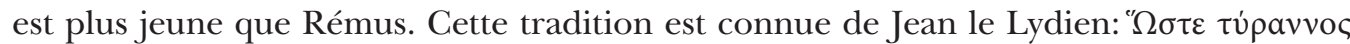

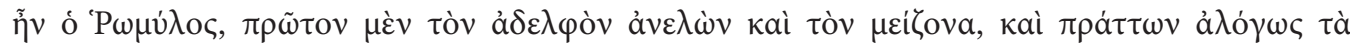

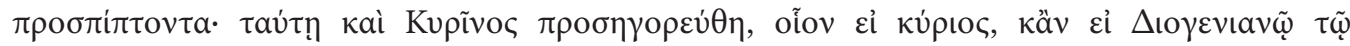

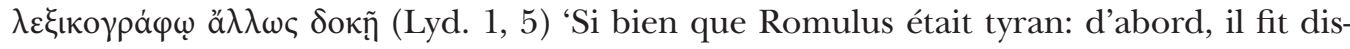
paraître son frère - qui était l'aîné, qui plus est -, et il traitait sans discernement les affaires qui se présentaient. C'est pourquoi il fut surnommé Quirinus, c'est-à-dire 'maître' [кúpıç] - même si le lexicographe Diogénien est d'un autre avis' (Dubuisson \& Schamp 2006: p. 12). À première vue, une tradition aussi tardive (Jean le Lydien écrit au VI siècle) pourrait inspirer la méfiance. Mais cette défiance s'atténue ${ }^{14}$ quand on observe que le mot altellus, dont la meilleure interprétation semble être 'le petit deuxième', se concilie parfaitement avec la présentation de Rémus comme le plus 'grand' des deux

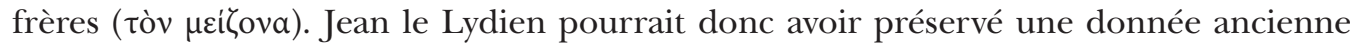
de l'histoire des jumeaux.

Il existerait donc à Rome une tradition qui faisait de Rémus le premier-né des jumeaux (celui qui est sorti du ventre de sa mère en premier). Le mot altellus ferait référence au statut de Romulus comme le deuxième des jumeaux (celui qui est venu au monde en second). Nous dirons que Rémus est l'aîné des jumeaux et Romulus le cadet (Carandini 2006: pp. 287, 379; Ver Eecke 2008: p. 198; Briquel 2013: p. 79; cf. Viarre 2005: p. 183, note 169). Il serait donc possible que le suffixe de *alter-(e)lo-s ait un sens diminutif (Carandini 2006: p. 86). Néanmoins, le suffixe -elo-s n'a pas obligatoirement une valeur diminutive (cf. aemulus). D'Alessio (2006: p. 475) désigne Rémus comme '[i]l primo gemello partorito'.

Il en résulte un intéressant paradoxe. Le conditor de Rome n'est pas l'aîné des jumeaux, mais le cadet (altellus). Cette conclusion pourrait paraître aberrante, mais il existe un parallèle remarquable, fourni par l'Ancien Testament: Jacob a pour jumeau Ésaü, Ésaü est le premier-né (Gn. 25, 25-26), et pourtant c'est Jacob qui deviendra (par ses fils) le fondateur d'Israël. Le statut de cadet que nous postulons pour Romulus doit donc être pris au sérieux en dépit de (ou plutôt en raison de) son caractère inattendu.

\section{La primauté paradoxale et momentanée de Rémus: la récupération du bétail volé}

Romulus et Rémus sont nés d’un seul et même géniteur, qui était, selon les versions, le dieu Mars ou une divinité masculine du foyer (Promathion, cité par Plutarque, Rom. 2, 4-5) ou un humain (Meurant 2000: p. 63). Les jumeaux albains ne sont donc pas comparables aux couples formés par Pollux (qui a un père divin, Zeus) et Castor (dont le père est le mortel Tyndare) ou par Héraclès (fils de Zeus) et Iphiclès (fils du mortel Amphitryon).

14 Sur ce passage et son orientation anti-romuliste et même anti-quiriniste (Kupĩvoc étant expliqué par le mot grec кúpıs), échos de batailles idéologiques de la République finissante, voir Ver Eecke (2008: p. 387). Cette orientation est précisément un gage de l'authenticité de l'information. 
À leur naissance, les jumeaux enfantés par Rhéa Silvia (ou Ilia) se distinguent de tous les autres bambins. Plutarque, dont les sources sont Dioclès de Péparéthos $(\Delta$ เок $\lambda \tilde{\eta} \varsigma$

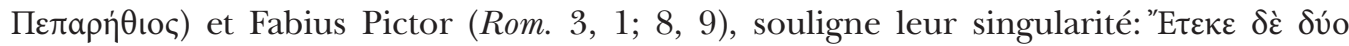

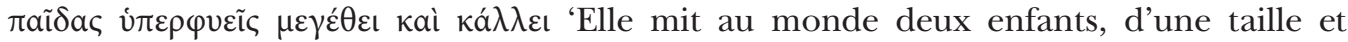
d'une beauté extraordinaires' (Plut. Rom. 3, 4). En grandissant, les jumeaux conservent

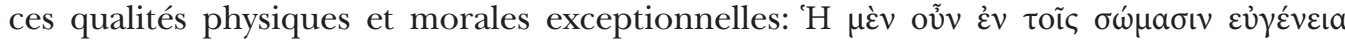

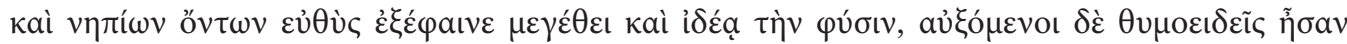

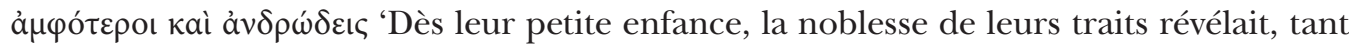
ils étaient grands et beaux, celle de leur nature; en avançant en âge, ils devinrent tous les deux résolus et courageux' (Plut. Rom. 6, 3). Romulus et Rémus mènent conjointement une vie agreste, parmi les pasteurs.

Mais une inégalité se dessine entre les deux frères. Selon les auteurs, c'est l'un ou l'autre des jumeaux qui a la préséance. Dans la tradition la plus répandue, Romulus est par certains aspects supérieur à son frère, comme cela est attendu pour le futur fondateur

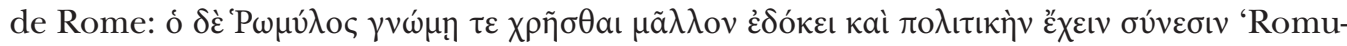
lus paraissait supérieur (à son frère) en réflexion et en intelligence politique' (Plut. Rom. 6, 3); perhibetur ut adoleuerit et corporis uiribus et animi ferocitate tantum ceteris praestitisse ut omnes qui tum eos agros ubi hodie est haec urbs incolebant aequo animo illi libenterque parerent $^{15}$ (Cic. Rep. 2, 2, 4). Dans la suite de l'histoire, Rémus subira trois échecs. D'abord,

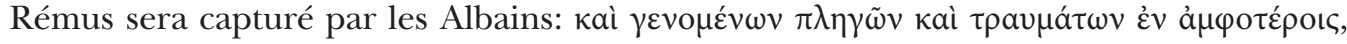

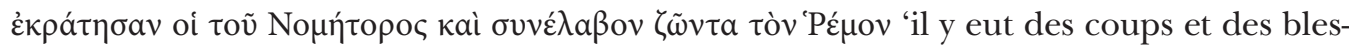
sures de part et d'autre, mais les hommes de Numitor eurent le dessus et firent Rémus prisonnier' (Plut. Rom. 7, 2). Ensuite, les auspices désigneront Romulus, non Rémus. Enfin, Rémus sera tué par son frère.

Ovide, qui tâche de réhabiliter Romulus, ${ }^{16}$ raconte que la vestale Silvia vit en songe deux palmiers (duae [...] palmae), dont l'un s'élevait plus haut que l'autre (ex illis altera maior erat), étendait ses lourds rameaux sur le monde entier (grauibus ramis totum protexerat orbem) et atteignait les astres par sa frondaison (contigeratque sua sidera summa coma, Ov. Fast. 3, 31-34). Selon Ovide, la supériorité de Romulus était visible dès le moment de la naissance: l'un des deux poupons aurait été plus vigoureux que l'autre (plus tamen ex illis iste uigoris habet, Ov. Fast. 2, 396). En réalité, cette différenciation entre les jumeaux dès leur petite enfance imaginée par Ovide ne reflète certainement pas un état ancien de la légende. Au contraire, Dasen (2005: p. 141) souligne que '[d]ans les arts figurés, Romulus et Rémus sont essentiellement deux nouveau-nés identiques'. ${ }^{17}$ La supériorité de Romulus ne devait apparaître qu'à l'âge mûr.

15 'Quand il [Romulus] fut devenu grand, il fit preuve, dit-on, d'une telle supériorité sur les autres, tant par sa force physique que par la fougue de son caractère, que tous les habitants des terres où se trouve aujourd'hui notre ville acceptèrent volontiers de lui obéir'. La référence aux uiribus est due au rapprochement de Rōmulus avec $\dot{\rho} \omega \dot{\mu \eta}$.

16 Schilling (1960: p. 189), Bruggisser (1987: pp. 91-93), Ver Eecke (2008: p. 466). Il fallait faire oublier le fratricide, la fin de règne tyrannique de Romulus, la tentative de couronnement de César lors des Lupercālia.

17 Cela n'exclut pas que l'un des jumeaux (mais pas l'autre) soit dès sa naissance prédestiné à un avenir éclatant. En ce sens, Carandini (2006: pp. 25, 266, 380, 551). 
Cette précellence attribuée à Romulus n'est pas surprenante et s'explique par le prestige qui entourait le fondateur de Rome (en dépit du malaise suscité par le fratricide). Néanmoins, Plutarque, qui souligne les aptitudes de Romulus, reconnaît aussi des qualités à son frère. Numitor est impressionné par la prestance de Rémus au moment où

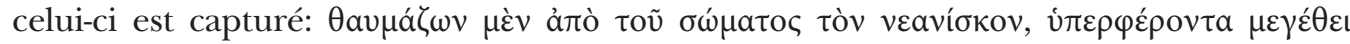

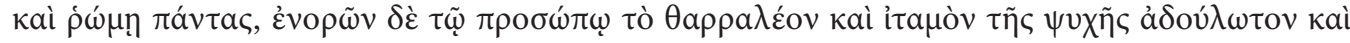

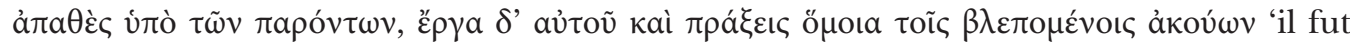
frappé par le physique du jeune homme, qui surpassait tout le monde en taille et en force, et observa que son visage respirait la résolution et la hardiesse et qu'il restait impassible et ne se laissait pas dominer par la situation; ce qu'il avait appris de sa conduite et de ses actes répondait à ce qu'il voyait' (Plut. Rom. 7, 5).

Il existe un témoignage textuel où la supériorité de Rémus sur Romulus est indéniable: le passage des Fastes d'Ovide concernant l'institution des Lupercales (Ov. Fast. 2, 361380). ${ }^{18}$ Quand les prêtres préparent la fressure enfilée sur des broches de saule (dumque sacerdotes ueribus transuta salignis / exta parant, 363-364), les jumeaux, qui s'adonnaient dévêtus à des activités sportives (corpora nuda, 366), apprennent que des brigands (praedones) sont en train de dérober leurs bouvillons (iuuencos). Ils poursuivent les voleurs en s'élançant dans des directions opposées (diuersis exit uterque / partibus, 371-372). Rémus les rejoint et récupère le butin (occursu praeda recepta Remi, 372). À son retour (ut rediit), grisé par le succès, Rémus enlève les exta qui grésillent sur les broches (ueribus stridentia detrahit exta, 373), en déclarant avec orgueil que personne ne les consommera sinon le vainqueur (atque ait: haec certe non nisi uictor edet, 374). Rémus dévore les exta et les Fabii l'imitent (Fabiique simul, 375). La réaction de Romulus est double. D'une part, il rit (risit, 377), et il faudra s'interroger sur la signification de ce rire. D'autre part, Romulus reconnaît la victoire de Rémus et des Fabii, et il s'afflige de sa propre défaite (indoluit Fabios potuisse Remumque / uincere, 377-378).

La mention de la supériorité du Rémus ovidien (uincere, 378) sur Romulus est trop étrange et paradoxale pour être analysée comme une invention d'Ovide ou une création récente de la tradition, puisque Ovide tend au contraire à valoriser le futur conditor de Rome. Acilius (dans Plut. Rom. 21, 9; fr. 3 de Chassignet 1996: p. 63) et Servius (ad Aen. 8, 343), qui narrent le même épisode, ne mentionnent pas l'infériorité de Romulus, peut-être parce qu'elle devait paraître incongrue (Briquel 2018: p. 137-138). L'authenticité du thème de la vélocité de Rémus est corroborée de façon indirecte par un fragment d'Aelius Tubero (fr. 4 de Beck \& Walter 2004: p. 350-352) cité par Denys d'Halicarnasse

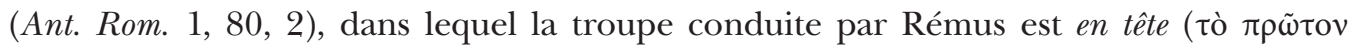

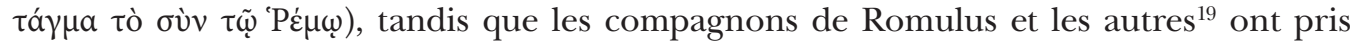

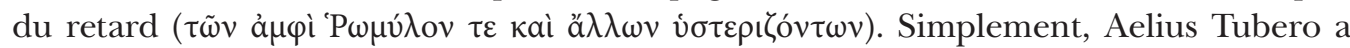
modifié la logique primitive du récit, puisque le groupe qui a de l'avance tombe dans une embuscade et Rémus est fait prisonnier, tandis que Romulus est épargné (Ver Eecke 2008: p. 474; 2010: p. 33; voir aussi Poucet 1985: p. 211). L'avance de Rémus, qui faisait

18 Texte, traduction et commentaire dans Schilling (1993a: pp. 42-43, 125-126).

19 Par anachronisme, Aelius Tubero fait allusion à la confrérie des luperci Iulii instituée en l'honneur de César. 
sa supériorité, est donc devenue une source d'infériorité dans l'œuvre d'Aelius Tubero. On conclura que la primauté de Rémus dans la narration ovidienne reflète une donnée ancienne de l'histoire des jumeaux albains et doit donc avoir un sens précis.

\section{Rémus s'enferme dans l'univers pré-civilisé patronné par Faunus}

La récupération par Rémus du bétail volé lui confère un succès éphémère. Mais Rémus gâche sa préséance en dévorant les exta. La scène de la consommation des exta annonce un retournement de la situation. Cette manducation des exta est susceptible d'une triple lecture. Primo, la consommation des exta s'interprète comme une usurpation de la part qui doit revenir à la divinité, donc comme un sacrilège, selon Schilling (1960: p. 192) qui compare Mézence (Mezentius) réclamant les prémices de la vendange (proxima musta, Ov. Fast. 4, 888) comme prix de la victoire (uincere, 889). ${ }^{20}$ Les hommes doivent se contenter des uiscera. Secundo, la manducation des exta représente une tentative d'appropriation des forces inhérentes aux exta. Or les exta étaient conçus comme le siège de la vie qu'on offrait aux divinités. Donc manger les exta signifie capter la vitalité que les exta renferment. ${ }^{21}$ Tertio, en s'arrogeant la part de Faunus, Rémus se condamne irrémédiablement à rester prisonnier du domaine patronné par Faunus, à savoir la nature non domestiquée et pré-civilisée. ${ }^{22}$ Cette sujétion au monde pré-urbain de Faunus interdira à Rémus de devenir le fondateur de Rome. Désormais, Rémus ne pourra plus quitter le monde des bergers-brigands.

Parce qu'il a ingéré les exta destinés à Faunus, une affinité marquée est apparue entre la personnalité de Rémus et les valeurs célébrées lors des Lupercālia, qui représentent une négation de la société civilisée dont la cité est le soubassement. Les luperques sacrifiaient des caprins (Val. Max. 2, 2, 9), femelles (capella, Ov. Fast. 2, 361; aĩ 1 ac, Plut. Rom. 21, 6) ou mâles (caprum, Ov. Fast. 2, 445; hircus, Ov. Fast. 2, 441: dans sacer hircus inito, le verbe est implicitement mis en relation avec le nom Inuus de Faunus; étymologie de Lupercalia comme LUere PER CAprum chez Quintilien 1, 5, 66, cf. l'écho affaibli spelunca, in qua de CApro LUebatur [...] unde et LU perCAl non nulli dictum putant, chez Serv. Aen. 8,343 , avec un jeu supplémentaire sur speLUnCA). Boucs et chèvres semblent avoir été associés à une forme de sauvagerie et symbolisaient l'élevage lointain dans une nature souvent montagneuse et peu apprivoisée. ${ }^{23}$ De surcroît, les luperques découpaient la peau des caprins pour confectionner des lanières et au moment de la course, ils étaient

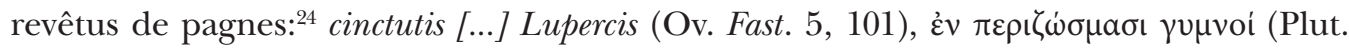

20 L'ingestion des exta n'est pas nécessairement un délit en soi. Ainsi, les Potitii, arrivés avant les Pinarii à l'appel d'Hercule, ont reçu le droit de consommer les exta (Liv. 1, 7, 12-14), mais le contexte est 'grec'. Sur les Potitii, voir Briquel (1980: pp. 273-274, 286-287, 291; 2018: pp. 140-141), Di Fazio (2013: p. 239).

21 Schilling (1960: p. 192). Définition du terme exta dans Schilling (1993b: p. 120, note 143).

22 Nous rappelons que Faunus a été mis en relation avec le personnage nommé 'Aүpıç, présenté comme le

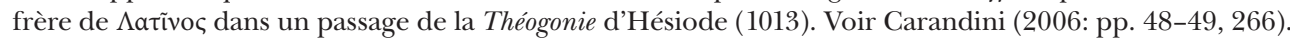

23 Vé (2018: p. 158). Sur ce motif, voir Ovide (Fast. 4, 511) et Apulée (Met. 8, 19, 1).

24 Les luperques n'étaient pas complétement nus. Voir Porte (1976), Ver Eecke (2008: p. 469, note 122), Vé 
Rom. 21, 7). Les luperques étaient 'ceints par pudeur des peaux des victimes qui venaient

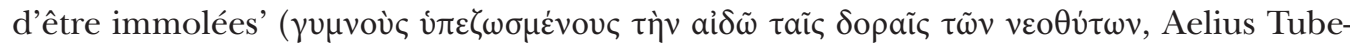
ro dans Dion. Hal. Ant. Rom. 1, 80, 1). En revêtant ces pagnes, les luperques quittaient symboliquement la société civilisée et manifestaient leur passage dans le monde sauvage.

Si l'existence d'un lien sémantique entre les mots lupercus et lupus 'loup' n'était pas illusoire,${ }^{25}$ les luperques seraient liés au loup qui est le symbole par excellence du monde sauvage. ${ }^{26}$ Une confrontation des luperques avec les Hirpī Sōrānī a été entreprise par plusieurs chercheurs. ${ }^{27}$ Selon une étiologie de Servius (ad Aen. 11, 785), lors d'un sacrifice à Dis Pater (cum aliquando Diti patri sacrum persolueretur) accompli sur le mont Soracte, des loups apparurent soudain et emportèrent du feu les exta (subito uenientes lupi exta de igni rapuerunt). Les bergers (pastores) pourchassèrent les loups et arrivèrent à une grotte d'où sortait une vapeur toxique (halitum [...] pestiferum) qui se révéla mortelle. La pestilence était apparue parce qu'ils avaient poursuivi les loups (exinde est orta pestilentia, quia fuerant lupos secuti). Un oracle proclama (de qua responsum est) que la pestilence pouvait se dissiper (posse eam sedari) s'ils imitaient les loups (si lupos imitarentur), c'est-à-dire s'ils vivaient de rapines (id est rapto uiuerent). Ils furent alors appelés Hirpi Sorani, car les loups sont désignés par le mot hirpi dans la langue des Sabins (nam lupi Sabinorum lingua uocantur hirpi). Ici, les bergers sont invités à se comporter comme des loups. Les Hirpi Sōrān̄ mimaient-ils le vol des exta et consommaient-ils ces exta? Cela serait concevable, mais le texte ne le dit pas explicitement. Le point important pour notre propos est que l'appropriation des exta est conçue comme un acte qui s'apparente aux agissements du loup (qu'il s'agisse du loup animal ou d'humains mimant cet animal) et qui relève donc d'un comportement typique du monde sauvage. Les exta sont mentionnés à propos du mont Soracte par Silius Italicus (5, 178). Mais le témoignage de Servius doit être manié avec précaution, car la localisation erronée (Hirpinorum) suggère que Servius a incorporé des éléments sabelliques allogènes tirés de Varron ou de Caton (Di Fazio 2013: p. 252).

Le rire de Romulus est susceptible de plusieurs lectures, qui ne sont pas incompatibles entre elles. En premier lieu, une interprétation psychologisante a reconnu un rire jaune, visant à dissimuler sa déconvenue personnelle, son dépit, son mécontentement. ${ }^{28}$

(2018: p. 141, note 16, 161, note 145). L'adjectif nüdus (cf. Paul. Fest. 49, 18-21 L) signifie 'en petite tenue, habillé à la légère'.

25 Mais voir les réserves de Flobert (2014: pp. 169-170). En outre, l'ingénieuse idée d'une parenté linguistique entre Faunus et $\theta$ aṽvov glosé comme $\theta$ piov (Blumenthal 1930: p. 38; Binder 1964: pp. 82-83), sans être impossible, n'est pas claire dans les détails (voir Chantraine 1999, sous $\Theta a u ́ \lambda$ เoc) et reste, en toute rigueur, non démontrée. Flobert (2014: p. 177) envisage pour Faunus une étymologie alternative qui serait assurément possible. Nous ne prenons pas position ici. Nous pensons qu'il est préférable de ne pas employer cet argument étymologique dans notre raisonnement. De plus, l'assimilation au Pan Lycaeus des Arcadiens (Liv. 1, 5, 2) ne suffit pas en soi à prouver que le nom Faunus ait signifié 'loup' à l'origine. La question reste ouverte.

26 Pour le loup comme redoutable prédateur et symbole de la sauvagerie (mais qui a ailleurs des connotations martiales), voir Bremmer (1987: pp. 30-31).

27 Par exemple Briquel (1980: pp. 274-275, 281; 2018: pp. 141, 144), Vé (2018: pp. 175-176).

28 L'expression 'rire jaune' est employée par Schilling (1960: p. 193; 1993a: p. 126, note 94) et Ver Eecke (2010: p. 31, note 38). 
En second lieu, le rire de Romulus sert d'étiologie au rire des deux jeunes garçons

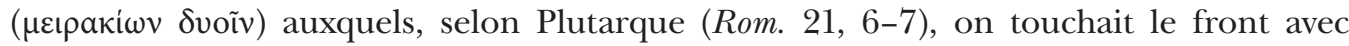

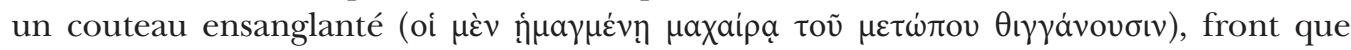

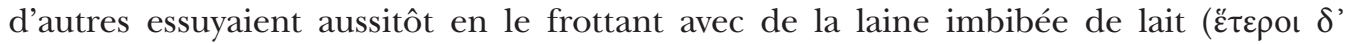

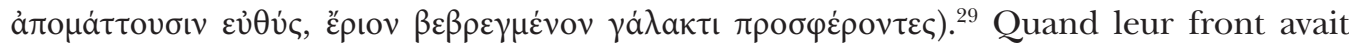

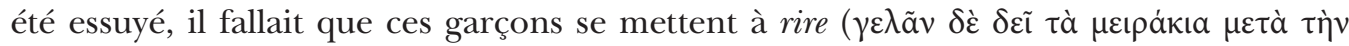
ảंó $\left.\mu \alpha \xi_{\imath} v\right)$. Un tel rire n'est pas exempt d'ambiguïté. Ce rire un peu fou (car sans cause apparente) s'interprète-t-il comme une renonciation temporaire (pour cette journée des Lupercālia) à la décence et à la bienséance (donc aux normes) qui encadrent la vie en société? Mais on rit aussi de l'indécence elle-même, car on sait que les comportements normés reprendront leurs droits à la fin de la journée. Ou plutôt le rire, à la fin du cycle annuel, est-il l'expression d'une socialisation retrouvée et ressourcée? Le rire ritualisé pourrait aussi avoir une fonction métacommunicative de signal, annonçant 'it is play'. ${ }^{30}$

En troisième lieu, le rire de Romulus rappelle le rire qui saisit Héraclès et Omphale (ridet et Alcides [...], ridet [...] Lyda puella, Ov. Fast. 2, 355-356) quand le libidineux Faunus a été jeté en bas du lit. Ici Faunus est privé d'un plaisir sexuel, tout comme là Faunus est privé des exta (car les exta étaient préparés pour Faunus). Dans le cas de la consommation des exta, le dieu qui patronne la subversion et l'anti-norme (Faunus) est lui-même victime d'un acte subversif de la part d'un mortel qui récuse la norme (Rémus).

En quatrième lieu, l'initiative inopinée de consommer les exta après une victoire facile, comme sur un coup de tête, fait de Rémus un être dangereusement imprévisible. Romulus prend conscience que Rémus est trop peu fiable pour fonder une cité.

En cinquième lieu, surtout, Romulus rit car il comprend que l'absorption des exta devrait interdire à Rémus de prétendre au rôle de leader civilisateur. Romulus, certes vaincu, pressent que, malgré le succès momentané de son frère, c'est lui-même qui recevra la première place dans l'avenir. Car Rémus aura la prééminence tant qu'il évoluera dans l'univers de Faunus, mais quand il s'agira de quitter le monde de Faunus pour fonder une cité, seul Romulus aura les aptitudes requises. ${ }^{31}$

\section{Pieux mais tricheur: I'ambiguiité de Romulus}

Les deux frères ne restent pas ensemble, mais se livrent à des activités différenciées. Romulus a un penchant pour les cérémonies et pour la divination. Il s'adonne à ces

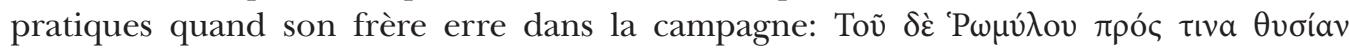

29 Le lait et la laine renvoient à la communauté pastorale et au monde rural. Le sang (image de la force vitale?) symboliserait Faunus, selon Vé (2018: pp. 159-160).

30 Cette fonction du rire a été mise en évidence (dans d'autres contextes) par Grammer \& Eibl-Eibesfeldt (1990).

31 Camous (2010: pp. 98, 162), auquel se réfère Briquel (2013: p. 87; 2018: p. 149), suggère que le rire en luimême représente un signe de civilisation. Certes, quelques mythes sud-américains étudiés par Lévi-Strauss (1964: pp. 101, 131, 140) évoquent un rire associé à l'invention culturelle. Mais ailleurs le rire a un effet désastreux. 


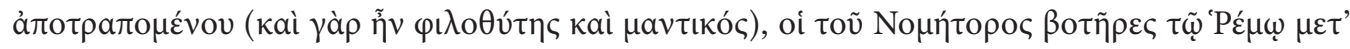

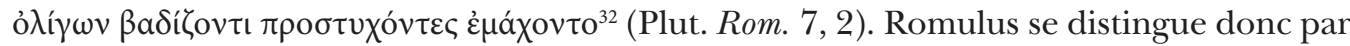
sa piété. La piété de Romulus contraste avec l'attitude désinvolte de Rémus, caractérisée par le rejet de la dichotomie fondamentale entre la part destinée aux hommes et la part réservée aux dieux (les exta).

Néanmoins, plusieurs sources évoquent un mensonge ou une tricherie dont Romulus se serait rendu coupable lors de la prise des auspices, au moment de fonder la ville. Selon Denys d'Halicarnasse (Ant. Rom. 1, 86, 3-4), sous l'effet de l'impatience et de la jalousie

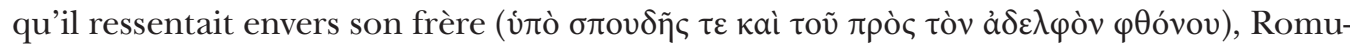
lus envoya des messagers à Rémus pour lui demander de le rejoindre avant même d'avoir

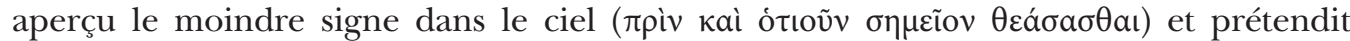

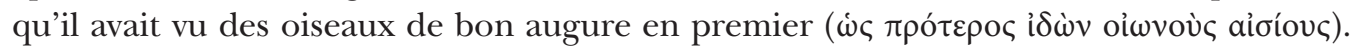

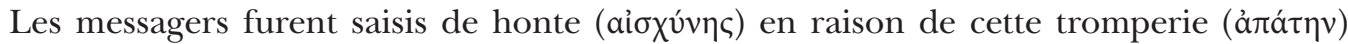
et ne se pressèrent pas. Alors, six vautours venant de la droite apparurent à Rémus ( $\tau \tilde{\omega}$

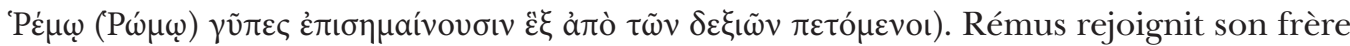

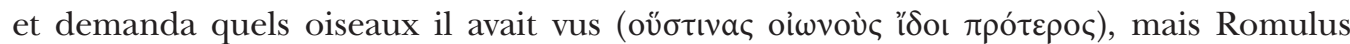

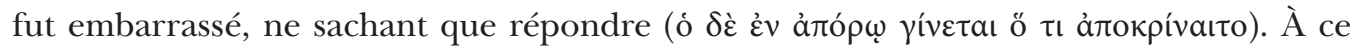

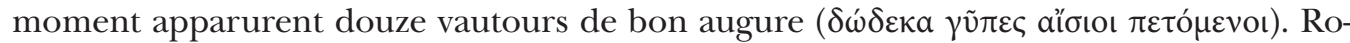
mulus s'adressa alors à Rémus en ces termes: 'pourquoi veux-tu savoir ce qui s'est passé

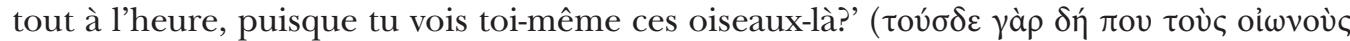

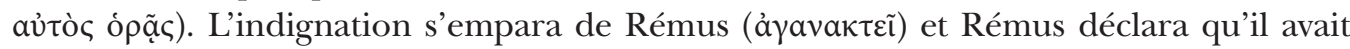

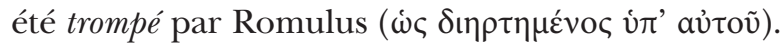

L'exposé de Plutarque (Rom. 9,5) est moins détaillé. Six vautours apparurent à Rémus

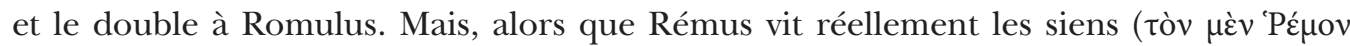

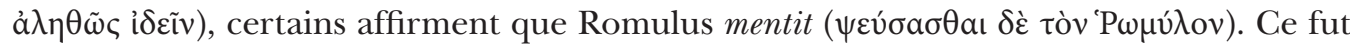

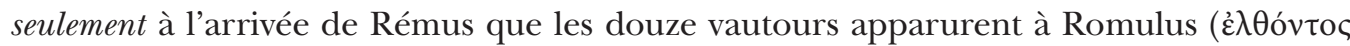

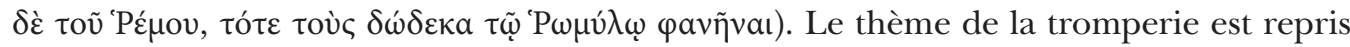

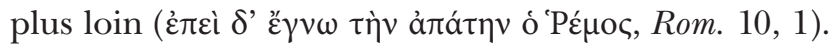

Cette curieuse accusation à l'encontre de Romulus doit être significative et refléter un aspect ancien de la légende et de l'idéologie qu'elle véhiculait. Nous ne pensons pas que la fourberie de Romulus s'explique simplement par la volonté des populārēs, qui se reconnaissaient en Rémus, d'instiller le doute sur la légitimité de Romulus (modèle de Sylla) à exercer le pouvoir et d'imputer à Romulus la responsabilité du conflit avec son frère, conflit qui serait le prélude à la première guerre civile.

$\mathrm{Si}$ on fait abstraction des aspects psychologisants qui auraient motivé la conduite de Romulus ( $\sigma \pi \circ \delta \delta \tilde{\eta} \varsigma, \varphi \theta$ óvov chez Denys), on doit admettre que, dans un état ancien de l'histoire, Romulus avait eu recours au mensonge. Cela semble rétrospectivement inutile, puisqu'il a obtenu douze vautours, et que la règle veut qu'un signe nouveau annule un

32 'Mais tandis que Romulus était retenu ailleurs par un sacrifice (car il aimait les cérémonies religieuses et pratiquait la divination), les bergers de Numitor, étant tombés sur Rémus qui cheminait avec quelques compagnons, engagèrent la bataille.' 
signe précédent. ${ }^{33}$ L'annonce prématurée de Romulus vient de la confiance qu'il a en son élection, car il sait que Rémus s'est disqualifié.

On fera contraster l'intelligence rusée (voire la fourberie) de Romulus avec la lourdeur d'esprit que l'un des Livres Pontificaux (libro Pontificalium secundo) cité par l'Origo Gentis Romanae $(22,2)$ attribue à Rémus. Tombant dans un piège en l'absence de Romulus (tum Romulus aberat), Rémus, confiant en sa force (fiducia uirium), est assez naïf pour consentir à se laisser lier les mains dans le dos (manibus post terga ligatis) afin de montrer qu'il est capable de soulever une pierre avec les dents (lapidem [...] mordicus sublatum) pour la porter le plus loin possible. Alors que Rémus fait une démonstration abusive de sa force, Romulus fait une démonstration abusive de son intelligence rusée. Et on constate que l'abus de ruse a plus de succès que l'abus de force. Rémus souffre donc d'une double tare, qui réside dans son comportement pré-civilisé et dans sa naïveté. Ces deux défauts font de Rémus un être arriéré et le condamnent d'avance à rester en-deçà du monde de la cité. En franchissant la ligne pomériale pour narguer Romulus, Rémus ne commet pas seulement un délit religieux: de façon plus grave encore, l'irruption de Rémus risquait d'introduire dans la polis des valeurs contraires à la civilisation, à la pondération et à l'intelligence que requiert la vie dans la cité. Ce risque explique et justifie son meurtre par Romulus.

\section{Premier-né et cadet du couple gémellaire: Rémus / Romulus, Ésaü / Jacob}

Une nouvelle question se pose. Existe-t-il un rapport de cause à effet entre le caractère pré-civilisé et naîf de Rémus et le fait que Rémus soit le premier-né des jumeaux? Inversement, faut-il supposer un lien logique entre la condition de cadet de Romulus (si c'est bien ainsi qu'il faut comprendre altellus) et son destin de fondateur de l'Urbs? Dans la logique propre du récit, la réponse est positive. Selon notre interprétation de l'idéologie sous-jacente, il existe un rapport d'iconicité ${ }^{34}$ constructionnelle ou de diagrammaticité $^{35}$ entre l'âge relatif des jumeaux et leur tropisme respectif vers la stagnation ou vers le progrès. Le premier-né des jumeaux, donc le plus 'âgé', représente un stade culturel tourné vers le passé pré-civilisé, avec un comportement empreint de naïveté et fondé sur la force brute. En revanche, le cadet des jumeaux, donc le plus jeune par la naissance,

33 Minora enim auguria maioribus cedunt nec ullarum sunt uirium, licet priora sint (Serv. Ecl. 9, 13); nam si dissimilia sint posteriora, soluuntur priora (Serv. Aen. 2, 691); in auguriis prima posterioribus cedere (Serv. Aen. 12, 183). Voir Briquel (2013: p. 89).

34 Le terme d'iconicité désigne une relation de similarité ou d'analogie entre signans et signatum (Kilani-Schoch \& Dressler 2005: pp. 39-40).

35 Un diagramme 'reproduit les propriétés configurationnelles de l'objet auquel il renvoie' (Kilani-Schoch \& Dressler 2005: p. 46). La diagrammaticité instaure une 'homologie proportionnelle' entre les relations des parties du signe (ici la différence de priorité dans la naissance) et les relations des parties du concept (ici les tropismes respectifs vers l'avenir et vers le passé du plus 'jeune' et de l'aîné). La logique de l'histoire des jumeaux (à Albe et chez les Hébreux) consiste à accorder le statut de signe à la priorité relative des naissances. 
représente un stade culturel plus évolué, tourné vers l'avenir, mobilisant les ressources de la $\mu \tilde{\eta} \tau c^{36}$ et permettant d'aboutir à la fondation d'une nouvelle société.

Cette hypothèse peut-elle être corroborée par d'autres considérations? La réponse sera positive, si on accepte de confronter l'histoire de Romulus et de Rémus à celle des jumeaux Jacob et Ésaü, en s'appuyant non seulement sur le récit de la Genèse (cf. de Pury 2006), mais également sur deux écrits intertestamentaires, le Livre des Jubilés ${ }^{37}$ et le Testament de Juda inclus dans les Testaments des douze patriarches. ${ }^{38}$ Dans ces deux textes, le premier-né Ésaü est tué par Jacob, son jumeau cadet. La pertinence de la comparaison a été mise en lumière par Carandini (2006: p. 286-287) et Carafa (2006: p. 450) qui écrit: 'Vi è un caso che ricorda il mito di Roma sia perché riguarda gemelli conflittuali sia perché un gemello viene ucciso sulle mura. Esaù è infatti il personaggio che mostra le più strette affinità con Remo. Egli è il primo nato, selvaggio ed empio, ed è ucciso da Giacobbe perché, dopo un accordo sull'eredità, aveva violato i confini della Palestina e, secondo una variante del mito non accolta nell'Antico Testamento, aveva tentato di superare le mura costruite dal gemello.' Nous précisons que cette histoire n'est pas simplement copiée sur celle de Romulus et Rémus. En outre, l'attestation relativement tardive de la tradition mineure reflétée dans les écrits intertestamentaires $n$ 'implique pas que cette tradition soit une création récente. La piste ouverte par Andrea Carandini et Paolo Carafa a été approfondie par Briquel (2013). De nombreuses homologies précises entre les deux couples de jumeaux sont repérables.

- Homologie $\mathrm{n}^{\circ} 1$. Jacob et Romulus sont destinés à devenir des fondateurs.

- Homologie $n^{\circ} 2$. Les entités fondées par Jacob et Romulus seront divisées en tribus. Grâce aux fils (et petits-fils) de Jacob, les douze tribus d'Israël verront le jour. ${ }^{39}$ Grâce à Romulus, Rome sera divisée en trois tribus, au sens originel du mot latin tribus, issu de ${ }^{*}$ trid $^{h} u s<*$ tri- $^{h} h_{1}{ }_{1} u-s$ et signifiant 'division en trois' ${ }^{40}$

- Homologie n ${ }^{\circ}$ 3. Il existe un lien entre les noms propres des fondateurs et les noms des entités politiques fondées. Jacob prend le nom d'Israël $(G n$. 32, 29). Le nom de Rōmulus est dérivé du nom Rōma.

- Homologie $\mathrm{n}^{\circ} 4$. Jacob et Romulus ont chacun un frère jumeau. Ésaü est le jumeau de Jacob, Rémus est le jumeau de Romulus.

- Homologie $n^{\circ} 5$. Au sein de leur couple gémellaire respectif, Jacob et Romulus sont les cadets, tandis qu'Ésaü et Rémus sont les aînés. Ésaü est sorti le premier du ventre de sa mère Rébecca $(G n$. 25, 22-26). Rémus est l'aîné de Romulus, comme le suggèrent

36 Sur la notion de $\mu \tilde{\tau} \tau \iota$, voir maintenant Govers Hopman (2012: pp. 24, 33, 37-39, 43, 71).

37 Le texte éthiopien a été édité par VanderKam (1989a). Traductions dans Caquot (1987) et VanderKam (1989b). Caquot \& Philonenko (1987: pp. Lxxiv-Lxxv) datent la composition des Jubilés du règne de Jean Hyrcan, tandis que VanderKam (1989b: p. vi) date la rédaction entre 170 et 150 avant notre ère.

38 Traduction dans Philonenko (1987). Caquot \& Philonenko (1987: p. Lxxxi) datent la composition des Testaments des douze patriarches de la seconde moitié du Irer siècle avant notre ère.

39 Sur les douze tribus d'Israël, on consultera de Vaux (1973: pp. 19-65).

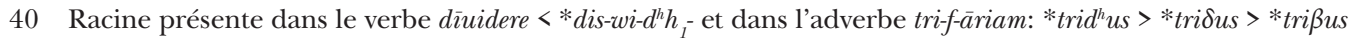
$>$ tribus (évolution phonétique $d^{h}>\delta>\beta>b$ au voisinage de $u$ comme dans iubeō 'ordonner'). Voir Weiss (2007), Martzloff (2019: p. 305) et la discussion de Brachet (2012). Sur les tribus romaines, voir Cels-SaintHilaire (1995). 
deux indices: d'un côté, la désignation de Romulus comme altellus 'le petit deuxième'; d'un autre côté, le témoignage (tardif, mais pouvant conserver ici une donnée ancienne) de Jean le Lydien.

- Homologie ${ }^{\circ} 6$. Le premier-né des jumeaux est peu éveillé intellectuellement. Ésaü n'a pas appris à écrire, à la différence de Jacob (Jub. 19, 13). Le naïf Rémus se laisse lier les mains sans aucune méfiance (Origo Gentis Romanae 22, 2).

- Homologie $n^{\circ} 7$. Le premier-né est caractérisé par une sorte d'animalité. Ésaü est velu comme une bête. Rémus a des accointances avec les luperques et s'assimile à Faunus en dévorant les exta qui lui étaient destinés.

- Homologie $\mathrm{n}^{\circ} 8$. Le plus jeune des jumeaux incarne un stade de développement plus avancé de l'Histoire de l'Humanité, tandis que l'aîné symbolise un état plus primitif (Briquel 2013: pp. 82, 88; 2018: pp. 189-190). Dans l'idéologie qui a présidé à la constitution du récit, ce stade jugé plus primitif est dévalorisé. Le cadet Jacob a une activité de pasteur-éleveur et d'agriculteur. Par contraste, l'aîné Ésaü est seulement un chasseur (Gunkel 1901: p. 271). Or un mode de subsistance fondé uniquement sur la chasse n'a pas d'avenir, comme le montre la scène où le malheureux Ésaü rentre bredouille, affamé et exténué $(G n .25,29-30)$ et doit donc se faire nourrir par son cadet. Jacob a certes un mode de vie nomade, mais Ésaü a un mode de vie qui peut être qualifié d'errant (ce qui est différent). De façon similaire, le cadet Romulus a de hautes préoccupations qui relèvent de la religion et de la divination. Tandis que Romulus s'y adonne, Rémus parcourt (cf. $\beta a \delta i \zeta o v \tau \iota)$ la campagne (Plut. Rom. 7, 2).

Nous pouvons donc observer une relation d'iconicité entre l'âge relatif des jumeaux et les phases successives de l'évolution des groupes humains. Le jumeau le plus 'jeune' symbolise le progrès et l'aspiration à un mode de vie civilisé (qui se matérialisent par une exploitation raisonnée des ressources de la nature dans le cas de Jacob, et par une organisation réfléchie de l'avenir au moyen des techniques auspiciales pour Romulus). Le jumeau le plus 'vieux' symbolise un mode de subsistance dépassé et peu civilisé (vie fondée sur les aléas de la chasse dans le cas d'Ésaü, vie de rapines fondée sur la force naïve dans le cadre d'une nature sauvage dans le cas de Rémus).

- Homologie n ${ }^{\circ}$. Celui des jumeaux qui est né le deuxième (Jacob, Romulus) jouit de la faveur divine.

- Homologie $\mathrm{n}^{\circ} 10$. La disqualification de l'aîné se manifeste en deux étapes: consommation des exta, et ensuite échec lors de la prise des auspices dans le cas de Rémus; renonciation au droit d'aînesse pour assouvir sa faim, et ensuite bénédiction manquée dans le cas d'Ésaü.

- Homologie $\mathrm{n}^{\circ} 11$. La première disqualification de l'aîné advient dans une scène relative à son alimentation. L'aîné prend une nourriture qu'il n'aurait pas dû consommer. Ésaü cède son droit d'aînesse contre un plat de lentilles (Gn. 25, 30-34). En consommant les exta réservés à la divinité, Rémus montre qu'il est incapable de suivre un comportement normé (et cela le rend inapte à vivre dans le cadre normé qui sera celui de la cité).

- Homologie $n^{\circ}$ 12. La deuxième disqualification de l'aîné (associée à la qualification du cadet) provient d'une autorité supérieure symbolisée par une puissance paternelle. Isaac, le père, ne donne pas à Ésaü la bénédiction principale et Ésaü doit réclamer une 
bénédiction mineure comme lot de consolation ( $G n$. 27). Jupiter, qui patronne les auspices, est un dieu-père, comme son nom l'indique: le théonyme Iuppiter est tiré du vocatif *dyoupater $<*$ dyeu pater (la gémination [pp] est comparable à celle de littera issu de *leitera, cf. leiteras CIL I ${ }^{2}$ 583, xxxv; fermeture de [a] en [i] en syllabe intérieure ouverte, comme dans accipiō, perficiō face à capiō, faciō).

- Homologie $\mathrm{n}^{\circ} 13$. Le cadet, qui sera le fondateur, a employé une tromperie fondée sur la ruse pour se qualifier face à son frère (travestissement de Jacob, mensonge de Romu-

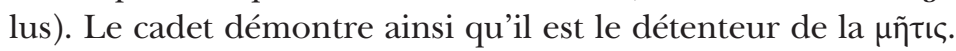

- Homologie $n^{\circ} 14$. Le jumeau écarté du processus de fondation se comporte en agresseur. Dans le Livre des Jubilés (37), les fils d'Ésaü, furieux de ce que leur père ait perdu son droit d'aînesse, le contraignent à prendre les armes contre son frère. Ésaü refuse d'abord, puis, par ressentiment envers Jacob, Ésaü se laisse convaincre et attaque la tour où Jacob est installé. Rémus franchit le sillon délimitant l'Urbs que Romulus a creusé.

- Homologie $n^{\circ} 15$. On assiste à la mise à mort de l'aîné agresseur par le jumeau qui jouit de la faveur divine et qui assure le rôle de fondateur. Dans le Livre des Jubilés (38), 'Jacob arma son arc, lança une flèche, transperça Ésaü, son frère, au sein droit et le tua' (Caquot 1987: p. 779; cf. Vanderkam 1989b: pp. 250-251). Dans le Testament de Juda (9, 3), 'Jacob frappa Ésaü d'une flèche, et il fut emporté blessé vers la montagne de Séir et mourut pendant le voyage, à Anoniram' (Philonenko 1987: pp. 861-862). Pareillement, Rémus est tué par Romulus (ou par un membre de l'entourage de Romulus, dans une version remaniée de l'histoire).

- Homologie n 16 . La mort de l'aîné n’a pas lieu immédiatement après la deuxième disqualification (bénédiction manquée, prise des auspices), mais plus tard, lorsque l'â̂né tente de pénétrer par la force dans l'espace où se tient le cadet (attaque de la tour par Ésaü, franchissement des limites de l'Urbs par Rémus). Voir Carandini (2006: pp. 287-288).

- Homologie $n^{\circ} 17$. L'enchaînement des épisodes converge vers la naissance d'un groupe nouveau et en fixe les revendications territoriales. Le cycle de Jacob et l'histoire de Romulus peuvent être décrits comme des légendes d'origine (de Pury 1991: pp. 86-88; 2006: p. 58).

Le nombre et la précision des homologies entre les deux couples gémellaires que nous avons énumérées permettent de conclure à l'existence d'une origine commune de la trame narrative. Inversement, l'examen systématique des homologies unissant les deux couples confirme que Romulus doit bien avoir le statut de cadet (statut identique à celui de Jacob), et corrobore donc l'interprétation de altellus comme 'le deuxième (des jumeaux)'. La comparaison confirme que le motif du fratricide est un élément narratif essentiel dans l'histoire des jumeaux albains.$^{41} A$ fortiori, ces homologies confirment le caractère relativement ancien du motif gémellaire dans la tradition sur les origines de Rome. ${ }^{42}$

41 Nous ne voulons pas nier toute valeur au rapprochement des jumeaux albains avec la légende de Пв $\lambda_{\text {Iá }} \varsigma$

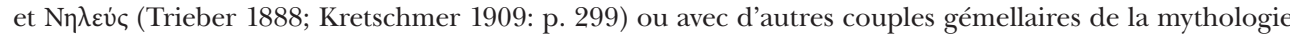
hellénique, mais nous soulignons que ces parallèles grecs ne possèdent qu'une pertinence limitée, car il leur manque l'épisode essentiel du fratricide.

L'existence d'un jumeau du fondateur de Rome n'est donc pas une 'invention' d'une tradition récente. 
Nous concluons qu'une même histoire mettant en scène des jumeaux aux tropismes divergents a dû circuler d'est en ouest, du Proche-Orient vers la péninsule italienne, et connaître des adaptations différentes dans ces deux aires culturelles éloignées. Il a été suggéré que Jacob incarnait les acquis de la révolution néolithique ${ }^{43}$ une mutation qui, au Proche Orient d'abord, fut le vecteur d'une économie promouvant l'agriculture et l'élevage, destinée à supplanter un mode de subsistance plus archaïque (et jugé périmé) qui serait fondé sur la chasse et la collecte. Acclimatée dans le Latium, cette histoire, croisée avec d'autres traditions narratives locales, a subi un décalage et a pris une connotation plus nettement politique, valorisant les comportements normés requis par la polis, une entité désormais érigée en modèle de civilisation. Par la suite, à Rome, la rivalité entre les jumeaux fut plusieurs fois réactualisée dans le cadre de différents conflits politiques, ce qui a conduit à de multiples réécritures de leur histoire.

\section{Conclusion}

Un passage de Jean le Lydien révèle l'existence d'une tradition qui considérait le futur conditor de Rome comme le cadet, tandis que son frère Rémus était le premier-né du couple gémellaire. L'authenticité et l'ancienneté de cette tradition sont confirmées par le mot altellus qui était cité dans le dictionnaire de Verrius Flaccus. Ce terme, déjà obscur pour les Romains eux-mêmes, s'interprète comme l'aboutissement de *alter-elo-s, un dérivé de alter désignant probablement celui des jumeaux qui était né en deuxième.

Une possible allusion cryptée à cette tradition se rencontre chez Ovide, qui en tire un effet littéraire: ex istis Romulus alter erit (Fast. 2, 386). Le poète augustéen était friand de ces jeux de mots fondés sur l'étymologie, comme le montre son admirable remotivation du théonyme Quirinnus (*ko-wir-ino-s) appliqué à Romulus divinisé: ante fuisse UIR $i$ coniunx nunc esse QUIRini (Met. 14, 834). Le fondateur de l'Urbs était donc bien le cadet. Pareillement, Jacob (le cadet), non Ésaü (l'aîné), devait devenir le fondateur d'Israël.

L'intérêt du mot altellus ne se limite pas à confirmer la pertinence du témoignage de Jean le Lydien. Le sémantisme de altellus condense en lui-même toute l'idéologie qui a présidé à la création de l'histoire de Romulus et Rémus (pour prendre les noms que les jumeaux portaient dans les versions classiques du récit). La désignation du futur conditor comme altellus transpose le rapport de diagrammaticité (iconicité constructionnelle) existant entre les âges relatifs des jumeaux et leurs tropismes respectifs vers un état primitif et révolu (le monde pré-civilisé, dont l'aîné Rémus ne pourra jamais sortir) ou vers l'avenir (le monde de la cité, incarné par le plus jeune, Romulus). Rémus, le plus vieux du couple gémellaire, symbolise un mode de vie dépassé, périmé, arriéré, destiné à être supplanté par les valeurs incarnées par le cadet novateur. La différence d'âge entre les jumeaux, certes minime, ${ }^{44}$ se voit amplifiée et dramatisée: elle est conçue comme la

43 En ce sens, explicitement, Briquel (2013: p. 82). On consultera, par exemple, Camps (1982: pp. 263-286).

44 Les jumeaux nartes naissent eux aussi à un intervalle, l'un au premier chant du coq (fyccag kerkuasæny), l'autre au second (dykkag karkuasany), quand paraît l'étoile du matin (Bonvarnony skastma). Le cadet est le plus brave. 
source de comportements antithétiques qui transposent à l'échelle de la vie individuelle deux stades successifs de l'évolution des communautés humaines.

La consommation des exta condamne Rémus non (ou non seulement) parce qu'elle constitue un acte sacrilège, mais plutôt parce que le refus de Rémus de se plier aux normes qui régissent le partage sacrificiel est interprétable comme l'indice d'une incapacité foncière de Rémus à se soumettre à toute forme de norme. Or une conduite normée est un prérequis de la vie au sein de la cité. Le rire de Romulus révèle l'intuition du cadet que désormais le rôle de fondateur ne pourra plus échoir à l'aîné. Dans cette logique implacable, le fratricide se comprend comme une nécessité qui s'impose au conditor (le plus jeune, conçu comme incarnant l'avenir, car détenteur de la $\mu \tilde{\eta} \tau \iota \varsigma)$ de bloquer tout risque d'irruption dans la polis des forces nocives émanant d'un univers perçu comme pré-civilisé et incarné par le jumeau plus vieux (le premier-né), trop attaché aux pesanteurs du passé et entièrement soumis aux aléas de la force brute.

\section{Bibliographie}

Beck, H., \& Walter, U. (2004). Die Frühen Römischen Historiker, II: Von Coelius Antipater bis Pomponius Atticus. Darmstadt: Wissenschaftliche Buchgesellschaft.

Billerbeck, M., \& Zubler, C. (2011). Stephani Byzantii Ethnica, II: $\Delta-I$. Berlin - New York: de Gruyter.

Billerbeck, M., \& Neumann-Hartmann, A. (2016). Stephani Byzantii Ethnica, IV: $\Pi-\Upsilon$. Berlin - Boston: de Gruyter.

Binder, G. (1964). Die Aussetzung des Königskindes Kyros und Romulus. Meisenheim: Hain.

Blumenthal, A. von (1930). Hesychstudien. Stuttgart: Kohlhammer.

Brachet, J.-P. (2012). Le tribūnus et le commandement d'un tiers de l'armée. Lucida Intervalla, 41, $5-34$.

Bremmer, J. N. (1987). Romulus, Remus and the Foundation of Rome. In J. N. Bremmer, \& N. M. Horsfall (Eds.), Roman Myth and Mythography (pp. 25-48). London: University of London.

Briquel, D. (1980). Trois études sur Romulus. In R. Bloch (Ed.), Recherches sur les religions de l'Antiquité classique (pp. 267-346). Paris - Genève: Droz; Champion.

Briquel, D. (2013). Deux histoires de jumeaux qui finissent mal: Romulus et Rémus, Jacob et Ésaü. Revue des Études Latines, 91, 67-94.

Briquel, D. (2018). Romulus, Jumeau et roi. Paris: Les Belles Lettres.

Bruggisser, P. (1987). Romulus Seruianus. Bonn: Habelt.

Camous, T. (2010). Romulus. Le rêve de Rome. Paris: Payot.

Camps, G. (1982). Introduction à la préhistoire. Paris: Perrin; Seuil.

Caquot, A. (1987). Jubilés. In A. Dupont-Sommer, \& M. Philonenko (Eds.), La Bible. Écrits intertestamentaires (pp. 627-810). Paris: Gallimard.

Caquot, A., \& Philonenko, M. (1987). Introduction générale. In A. Dupont-Sommer, \& M. Philonenko (Eds.), La Bible. Écrits intertestamentaires (pp. XV-CXLVI). Paris: Gallimard.

Carafa, P. (2006). La fondazione della città. In A. Carandini (Ed.), La leggenda di Roma, I: Dalla nascita dei gemelli alla fondazione della città (pp. 373-452). Roma: Fondazione Lorenzo Valla Milano: Arnoldo Mondadori. 
Carandini, A. (2006). Remo e Romolo. Dai rioni dei Quiriti alla città dei Romani (775/750-700/675 a. C. circa). Torino: Einaudi.

Cels-Saint-Hilaire, J. (1995). La République des tribus. Du droit de vote et de ses enjeux aux débuts de la République Romaine (495-300 av. J.-C.). Toulouse: Presses Universitaires du Mirail.

Chantraine, P. (1999). Dictionnaire étymologique de la langue grecque. Histoire des Mots. Avec un Supplément. Paris: Klincksieck.

Chassignet, M. (1996). L'annalistique romaine, I: Les Annales des Pontifes. L'annalistique ancienne (fragments). Paris: Les Belles Lettres.

Chassignet, M. (1999). L'annalistique romaine, II: L'annalistique moyenne (fragments). Paris: Les Belles Lettres.

D’Alessio, M. T. (2006). Fratelli/gemelli, tra cooperazione e conflitto. In A. Carandini (Ed.), La leggenda di Roma, I: Dalla nascita dei gemelli alla fondazione della città (pp. 469-476). Roma: Fondazione Lorenzo Valla - Milano: Arnoldo Mondadori.

Dasen, V. (2005). Jumeaux, jumelles dans l'Antiquité grecque et romaine. Zürich: Akanthus.

de Pury, A. (1991). Le cycle de Jacob comme légende autonome des origines d'Israël. In J. A. Emerton (Ed.), Congress Volume Leuven 1989 (pp. 78-96). Leiden - New York: Brill.

de Pury, A. (2006). The Jacob Story and the Beginning of the Formation of the Pentateuch. In T. B. Dozeman, \& K. Schmid (Eds.), A Farewell to the Yahwist? The Composition of the Pentateuch in Recent European Interpretation (pp. 51-72). Atlanta: Society of Biblical Literature.

de Vaux, R. (1973). Histoire ancienne d'Israël, II: La période des Juges. Paris: Lecoffre; Gabalda.

Di Fazio, M. (2013). Gli Hirpi del Soratte. In G. Cifani (Ed.), Tra Roma e l'Etruria. Cultura, identità e territorio dei Falisci (pp. 231-264). Roma: Quasar.

Dubuisson, M., \& Schamp, J. (2006). Jean Le Lydien. Des magistratures de l'État romain, I, 2: Introduction générale. Livre 1. Paris: Les Belles Lettres.

Flobert, P. (2014). Grammaire comparée et variétés du latin. Genève: Droz.

Govers Hopman, M. (2012). Scylla. Myth, Metaphor, Paradox. Cambridge - New York - Melbourne: Cambridge University Press.

Grammer, K., \& Eibl-Eibesfeldt, I. (1990). The ritualisation of laughter. In W. A. Koch (Ed.), Natürlichkeit der Sprache und der Kultur (pp. 192-214). Brockmeyer: Bochum.

Grandazzi, A. (1998). Lieu d'où l'on vient? Lieu où l'on va? De la Porta Romanula en particulier et des portes de Rome en général. In M. Humbert, \& Y. Thomas (Eds.), Mélanges de droit romain et d'histoire ancienne. Hommage à la mémoire de André Magdelain (pp. 175-195). Paris: Panthéon-Assas.

Gunkel, H. (1901). Genesis übersetzt und erklärt. Göttingen: Vandenhoeck \& Ruprecht.

Hagen, H. (1876). Glossographie. Jahresbericht über die Fortschritte der classischen Alterthumswissenschaft, 5, 338-355.

Kilani-Schoch, M., \& Dressler, W. U. (2005). Morphologie naturelle et flexion du verbe français. Tübingen: Narr.

Kretschmer, P. (1909). Remus und Romulus. Glotta, 1, 288-303.

Lachenaud, G. (2010). Scholies à Apollonios de Rhodes. Paris: Les Belles Lettres.

Lévi-Strauss, C. (1964). Mythologiques, 1: Le cru et le cuit. Paris: Plon.

Lhommé, M.-K. (2011). Trois auteurs, trois lexiques, trois visions de Rome. Verrius Flaccus, Pompeius Festus et Paul Diacre. In M. Simon (Ed.), Identités Romaines (pp. 129-143). Paris: Éditions Rue d'Ulm. 
Lindsay. W. M. (1891). Notes on Festus and Nonius. The Classical Review, 5, 9-11.

Lindsay, W. M. (1913). Sexti Pompei Festi De Verborum Significatu quae supersunt cum Pauli Epitome. Leipzig: Teubner.

Löwe, G. (1875). Quaestionum de glossariorum Latinorum fontibus et usu particula commentatio. Lipsiae: Teubner.

Martzloff, V. (2013). Review of P. Baldi, \& P. Cuzzolin (Eds.), New Perspectives on Historical Latin Syntax, 4: Complex Sentences, Grammaticalization, Typology (Berlin - New York 2011). Kratylos, 58, 115-129.

Martzloff, V. (2015-2016). La désignation des 'captifs' en albanais du Caucase (albanien), le verbe arménien gerem 'emmener en captivité' et le traitement phonétique de $* d^{h}$ intervocalique en arménien. Wék $k^{w}$ os, 2, 109-177.

Martzloff, V. (2019). La malédiction osque de Capoue à l'encontre de pakis kluvatiis (ST Cp 37, Vetter 6, Audollent 193). Wékwos, 5, 263-340.

Meurant, A. (2000). Romulus, jumeau et roi. Aux fondements du modèle héroïque. Revue Belge de Philologie et d'Histoire, 78(1), 61-88.

Nogara, B. (1916). Intorno al significato del nome di 'Roma'. Bullettino della Commissione Archeologica Comunale di Roma, 44, 141-144.

Peruzzi, E. (1967). Altellus. La Parola del Passato, 22, 367-368.

Philonenko, M. (1987). Testaments des douze patriarches. In A. Dupont-Sommer, \& M. Philonenko (Eds.), La Bible. Écrits intertestamentaires (pp. 811-944). Paris: Gallimard.

Porte, D. (1976). Note sur les 'luperci nudi'. In P. Gros, \& M. Gras (Eds.), L'Italie préromaine et la Rome républicaine. Mélanges offerts à Jacques Heurgon (Vol. II; pp. 817-824). Rome: École Française de Rome.

Poucet, J. (1985). Les Origines de Rome. Bruxelles: Facultés Universitaires Saint-Louis.

Prosdocimi, A. L. (2010). La Roma 'Tarquinia' nella lingua: forme e contenuti tra il prima e il dopo. Annali della Fondazione per il museo 'Claudio Faina', 17, 367-489.

Rocca, G. (2005). Etimologie italiche. In P. Biavaschi, G. M. Facchetti, \& G. Rocca (Eds.), Miscellanea italica (pp. 81-98). Milano: Arcipelago.

Schilling, R. (1960). Romulus l'élu et Rémus le réprouvé. Revue des Études Latines, 38, 182-199.

Schilling, R. (1993a). Ovide, Les Fastes, I: Livres I-III. Paris: Les Belles Lettres.

Schilling, R. (1993b). Ovide, Les Fastes, II: Livres IV-VI. Paris: Les Belles Lettres.

Secret, F. (1959). Egidio da Viterbo. Scechina e Libellus de Litteris Hebraicis. Roma: Centro Internazionale di Studi Umanistici.

Stein Kokin, D. (2011). Entering the labyrinth: On the Hebraic and Kabbalistic universe of Egidio da Viterbo. In I. Zinguer, A. Melamed, \& Z. Shalev (Eds.), Hebraic Aspects of the Renaissance. Sources and Encounters (pp. 27-42). Leiden - Boston: Brill.

Trieber, C. (1888). Die Romulussage. Rheinisches Museum, 43, 569-582.

VanderKam, J. C. (1989a). The Book of Jubilees. A critical text. Louvain: Peeters.

VanderKam, J. C. (1989b). The Book of Jubilees. Translation. Louvain: Peeters.

Vé, K. K. (2018). La cité et la sauvagerie: les rites des Lupercales. Dialogues d'Histoire Ancienne, 44(2), 139-190.

Ver Eecke, M. (2008). La République et le roi. Le mythe de Romulus à la fin de la République romaine. Paris: De Boccard. 
Ver Eecke, M. (2010). Ovide, conteur d'histoire: les Lupercales (Fastes II.267-452). Dialogues d'Histoire Ancienne, Supplément, 4(1), 25-41.

Viarre. S. (2005). Properce. Élégies. Paris: Les Belles Lettres.

Warren, M. (1884). On Latin Glossaries, with especial reference to the Codex Sangallensis 912. Transactions of the American Philological Association, 15, 124-228.

Weinstock, S. (1933). Tellus. Glotta, 22, 140-152.

Weiss, M. (2007). Cui Bono? The Beneficiary Phrases of the Third Iguvine Table. In A. J. Nussbaum (Ed.), Verba Docenti. Studies in historical and Indo-European linguistics presented to Jay H. Jasanoff by students, colleagues, and friends (pp. 365-378). Ann Arbor - New York: Beech Stave Press.

Wiseman, T. P. (1995). Remus. A Roman myth. Cambridge: Cambridge University Press.

Wiseman, T. P. (1998). Roman Drama and Roman History. Exeter: University of Exeter Press.

Vincent Martzloff / martzloffvincent@gmail.com

Institute of Latin Studies

Sorbonne University, Paris

1, rue Victor Cousin, 75005 Paris, France

Mgr. Barbora Machajdíková, Ph.D. / machajdikova.b@gmail.com

Department of Classical and Semitic Philology

Comenius University in Bratislava, Faculty of Arts

Gondova 2, 81102 Bratislava, Slovak Republic 